\title{
Multi-DOF Robotic Manipulator Trajectory Controlling Based on Minimum Energy Optimization
}

\author{
H. Du, J. M. Du, L. A. Chen, Z. W. Mai, X. H. Liu \\ College of Mechatronics and Control Engineering, \\ Shenzhen University \\ Shenzhen, Guangdong, P. R. China
}

\author{
H. Z. Cai \\ LK Leadwell Shenzhen Technology Co., Ltd. \\ Shenzhen, P. R. China
}

\begin{abstract}
This paper presents a methodology for determining an optimal trajectory based on minimizing energy consumption. Firstly we analyze a work process of a robotic manipulator between two given configurations at a given time, and the effect of main factors on motor energy consumption is also analyzed. A mathematical model of torque is used for obtaining a mathematical expression of each joint's torque and angle relationships. More specifically, for a multi-variable, higher order total torque model, a variational principle is used to obtain many differential equations for a multi-DOF (degrees of freedom) robotic manipulator, and iterative optimization is used to determine the optimal trajectory. Finally, a simulation shows that the optimal trajectory can save energy compared with two other trajectories.
\end{abstract}

Keyword-robotic manipulator; mathematical model; torque model; variational principle; energy consumption

\section{INTRODUCTION}

In recent decades, a large number of robotic manipulators have been used, greatly improving production efficiency. When repeated movements in industrial jobs are considered, the minimization of energy consumption will become significantly important [1]. In some cases, energy use minimization is considered as a sub-performance index, embedded in a multi-objective optimization problem [2]. Many scholars have studied this issue. James T. Allison proposed plant-limited co-design (PLCD) that is a design methodology for meeting new requirements at a minimum cost through limited physical systems [3], Wing Kwong Chung [4] designed and developed a novel genetic operator with an optimization goal of searching a path with a minimum energy demand, and Abhishek Agrawal proposed a design methodology which may allow a robot to approximately follow desired trajectories without actuator inputs [5]. Further, Amin Nikoobin [6] proposed a nonlinear disturbance observer to analyze the stability of a manipulator to get superior tracking performance. The dynamic characteristic of robot manipulators is uniquely determined by base parameters, which are functions of physical parameters [7]. The difficulty in planning manipulator trajectories lies in that nonlinear dynamic characteristics are highly coupled [8]. There are many ways to reduce energy consumption. D Meike [9] obtained energy-optimal trajectories by means of time scaling, and WF Faris [10] used a genetic algorithm to optimize unknown parameters of the trajectory for minimizing energy consumption. However, there is still no method to solve this problem from the perspective of optimizing a high-order torque equation. Therefore, this paper attempts to use a methodology for optimizing manipulator dynamics performance in order to find the best path solution.

\section{Robotic Manipulator Movement Depicted}

The main role of the robotic manipulator is to hold and move a work piece from one place to another. As fig. 1 shows, each joint is driven by its relevant motor. When the robotic manipulator has completely clamped a work piece, the work piece starts moving from position A to position B by rotating each joint motor. The robotic manipulator must complete this process within a predetermined period of time $0-t_{f}$. During this process, each of the manipulator's joint angle ranges from $\theta_{A i}$ to $\theta_{B i}(\mathrm{i}=1,2, \ldots \mathrm{n}$, where $\mathrm{n}$ denotes the DOF of the manipulator). Our mission is to reasonably plan each joint angle to minimize the manipulator energy consumption under this angle trajectory, so as to solve the actual energy consumption problems.

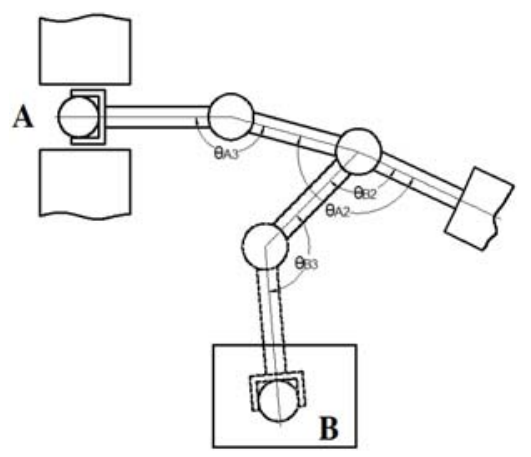

FIGURE I. Diagrammatic SKetch of Robotic ARm Initial Position A AND TERMINAL POSITION B.

\section{RoBOtic MANipUlator ENERGy CONSUMPTION FACTORS}

In order to achieve the minimum energy consumption in manipulator control, we take manipulator energy consumption factors being analyzed to create a function of the energy consumption $Q$. In consideration of a manipulator powered by electricity, the consumption of electricity is mainly in the operation of the motors, so we evaluate an excited DC servo motor for the study. We adopt an armature circuit voltage balance equation as eqn. 1 :

$$
u_{j}(t)=L_{j} \frac{d i_{j}(t)}{d t}+R_{j} i_{j}(t)+E_{j}(t)
$$


where $u_{j}, L_{j}, i_{j}, R_{j}$, and $E_{j}$ respectively represent the motor input voltage of an $i$-th joint, the inductance of the armature, the armature current, the counter electromotive force and armature resistance. The back EMF $E_{j}$ is proportional to the speed $w_{j}$ as in eqn. 2, and the calculation formula of electromagnetic torque $M_{j}$ is as in eqn. 3 :

$$
\begin{gathered}
E_{j}(t)=C_{0} w_{j}(t) \\
M_{j}=C_{m} i_{j}(t)
\end{gathered}
$$

where $C_{0}$ is an electric potential constant, and $C_{m}$ is a motor torque coefficient.

Newton's equations of motion can be obtained from the motor shaft torque balance equation eqn. 4 :

$$
T_{j \mathbf{0}}+f_{m} w_{j}(t)=M_{j}(t)
$$

where $T_{j 0}$ and $f_{m}$ respectively denote the motor shaft external load torque and viscous friction coefficient.

We assume that completion time of the entire operation process is $t_{f}$, and the electrical energy $Q_{j}$ which transforms into other forms of energy is calculated by eqn. 5 .

$$
Q_{\mathrm{j}}=\int_{0}^{t_{f}} P_{j}(\mathrm{t}) \mathrm{dt}=\int_{0}^{t_{f}} u_{j}(\mathrm{t}) i_{j}(\mathrm{t}) \mathrm{dt}
$$

We can obtain eqn. 6 by merging and simplifying eqn. 1 , eqn. 2 , eqn. 3 and eqn. 4 .

$$
Q_{\mathrm{j}}=L I_{j}^{2}\left(\mathrm{t}_{f}\right)-\mathrm{LI}_{j}^{2}(0)+\int_{0}^{t}\left(\mathrm{R}_{j} \mathrm{I}_{j}^{2}+\frac{C_{0} f_{m}}{C_{m}} \mathrm{w}_{j}^{2}\right) \mathrm{dt}+\frac{C_{0}}{C_{m}} \int_{0}^{t} T_{j 0} \mathrm{wdt}
$$

The start-up current $I_{j}(0)$, the termination current $I_{j}\left(t_{f}\right)$, the armature resistance $R_{j}$ and the viscosity friction coefficient $f_{m}$ are small values, and the main energy consumption of the motor is from the external load active torque. As seen from eqn. 6, the value of $Q_{j}$ predominantly relies on the last item.

\section{MAThematical ToRQue Model of Robotic MANIPULATOR}

To simplify the mathematical manipulator model and taking into account the inconsistency of morphological parameters of different robotic manipulators, in general, each manipulator arm will be considered to be a uniform quality connecting rod, and each connecting rod is driven by a corresponding motor, as shown in fig. 2.

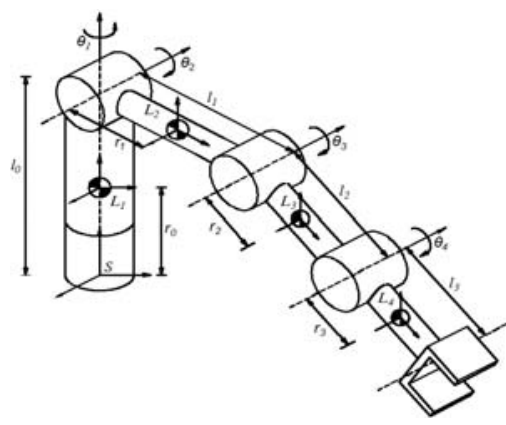

FIGURE II. SIMPLIFIED STRUCTURAL MODEL OF MULTI-DOF ROBOTIC MANIPULATOR.

To obtain the relationship between the motor torque and angle, we construct the Lagrangian expression eqn. 7:

$$
L(\theta, \dot{\theta})=T(\theta, \dot{\theta})-V(\theta)
$$

where the first term of the equation is system kinetic energy and the second is system potential energy.

$$
T(\theta, \dot{\theta})=\frac{\mathbf{1}}{\mathbf{2}}\left(V_{s l_{i}}^{b}\right)^{T} \psi_{i} V_{s l_{i}}^{b}
$$

Here, $V_{s l_{i}}^{b}$ represents the rotational speed of the $i$-th rod with respect to the coordinate system established at the intersection between a joint $i$-th rod and a joint $(i-1)$-th rod, and its value is calculated by eqn. 9. Here, $\psi_{i}$ denotes an inertia matrix for the $i$-th joint, and a diagonal matrix value is determined by eqn. 10 :

$$
V_{s l_{i}}^{b}=J_{i}(\theta) \dot{\theta}
$$

$$
\Psi_{\mathrm{i}}=\left[\begin{array}{llllll}
m_{i} & & & & & \\
& m_{i} & & & & \\
& & m_{i} & & & \\
& & & I_{x i} & & \\
& & & & I_{y i} & \\
& & & & & I_{z i}
\end{array}\right]
$$

where $J_{i}(\theta)$ is the body manipulator Jacobian for the $i$-th rod and $\theta$ is the generalized rotation angle of each joint. The rotational direction is changed by the rotation of the motor shaft, and its value can be calculated by eqn. 11 :

$$
\widetilde{\zeta}_{j}=e^{\zeta_{j} \theta_{j}} \cdots e^{\zeta_{i} \theta_{i}} \zeta_{j}
$$

where $\widehat{\zeta}_{j}$ denotes the rotation direction of a $j$-th shaft with respect to an $i$-th shaft after rotation. The body manipulator Jacobian $J_{s l_{i}}^{b}$ of the $i$ axes can be obtained by eqn. 12, where $\zeta_{i}^{+}$is the twist of the $i$ axis relative to the centroid coordinates of an $i$-th rod, as in eqn.13: 


$$
\begin{gathered}
J_{s l_{i}}^{b}(\theta)=\left[\zeta_{1}^{+}, \cdots, \zeta_{i}^{+}, \mathbf{0}, \cdots \mathbf{0}\right] \\
\zeta_{i}^{+}=\left[\begin{array}{c}
\zeta \times{ }^{l_{i}} L_{i} \\
\zeta
\end{array}\right]
\end{gathered}
$$

where ${ }^{l i} L_{i}$ denotes the coordinates of a point which passes through the $i$-th rotation axis with respect to the centroid coordinates of the $i$-th rod.

We can obtain the generalized torque of each joint by the Lagrange equation eqn. 14 [11].

$$
\frac{d}{d x} \frac{\partial L}{\partial \dot{\theta}}-\frac{\partial L}{\partial \theta_{i}}=\tau
$$

$M$ represents body manipulator Jacobian matrices multiplied by inertia matrices.

$$
M(\theta)=\sum_{i=1}^{n} J_{i}^{T} \psi_{i} J_{i}
$$

When we combine eqn. 7, eqn. 8, eqn. 9 and eqn. 15 and substitute the combination into eqn. 14 , eqn. 16 can be obtained.

$$
\tau_{i}=\sum_{j=1}^{n} M_{i j}(\theta) \ddot{\theta}_{j}+\sum_{j, k=1}^{n} \frac{\mathbf{1}}{\mathbf{2}}\left(\frac{\partial M_{i j}(\theta)}{\partial \theta_{k}}+\frac{\partial M_{i k}(\theta)}{\partial \theta_{j}}-\frac{\partial M_{k j}(\theta)}{\partial \theta_{i}}\right) \dot{\theta}_{j} \dot{\theta}_{k}+\frac{\partial V(\theta)}{\partial \theta_{i}}
$$

We can then select the base coordinate's plane for the system zero potential energy plane. System potential energy $V$ $(\theta)$ is expressed as eqn. 17 :

$$
V(\theta)=m_{1} g h_{1}(\theta)+\cdots+m_{n} g h_{n}(\theta)
$$

where $n$ represents the degree of freedom of the manipulator, and $h_{i}$ is the vertical distance between the centroid of the $i$-th rod and the zero potential energy surface. Total torque $J_{\tau}$ satisfies eqn. 18.

$$
J_{\tau}=\int_{0}^{t_{f}}\left[\tau_{1}(\mathrm{t})+\cdots+\tau_{n}(\mathrm{t})\right] d t
$$

Our goal is reasonable real-time planning of the joint angle, so that the total acting torque reaches a minimum at a specified time $t_{f}$.

\section{TOTAL TORQUE Minimization FUnCTIONAL EQUATION}

By substituting eqn. 16 into eqn. 18, we can obtain eqn. 19 related to acceleration, speed and angle of each joint angle:

$$
J_{\tau}=\int_{0}^{t_{f}} H\left(\theta_{1}(\mathrm{t}), \cdots, \theta_{\mathrm{n}}(\mathrm{t}), \dot{\theta_{1}}(\mathrm{t}), \cdots, \dot{\theta_{n}}(\mathrm{t}), \ddot{\theta_{1}}(\mathrm{t}), \cdots, \ddot{\theta_{n}}(\mathrm{t})\right) d t
$$

where $H$ represents a total torque per unit time, that is,
$H(t)=\tau_{1}(\mathrm{t})+\tau_{2}(\mathrm{t})+\tau_{3}(\mathrm{t})$, which is a functional equation regarding each joint angle. To get the total minimum torque per unit time, we can build a set of differential equations based on a functional variation law, that is, the Euler formula, as eqn. 20 shows:

$$
\begin{gathered}
H_{\theta_{1}(t)}-\frac{d}{d t} H_{\theta_{1}(t)}+\frac{d^{2}}{d x^{2}} H_{\theta_{1}(t)}=0 \\
\vdots \\
H_{\theta_{n}(t)}-\frac{d}{d t} H_{\theta_{n}(t)}+\frac{d^{2}}{d x^{2}} H_{\theta_{n}(t)}^{.}=0
\end{gathered}
$$

where $H_{\theta}$ represents an $H$ partial derivative for $\theta$, and we can obtain the expression of $H$ by substituting eqn. 16 into eqn. 18.

\section{SIMULATION}

In this paper, we take a three degrees of freedom robotic manipulator as a study object. We set a group of parameters (as show table 1) for the study to obtain the manipulator angle change image in real simulation time.

TABLE I. Manipulator Simulation Parameter SETtings

\begin{tabular}{|c|c|c|c|}
\hline$I_{x 1}=12.333$ & $I_{y 1}=12.1633$ & $I_{z 1}=0.4967$ & $\mathrm{Kg}^{*} \mathrm{~m}^{2}$ \\
\hline$I_{x 2}=4.908$ & $I_{y 2}=0.351$ & $I_{z 2}=5.043$ & $\mathrm{Kg}^{*} \mathrm{~m}^{2}$ \\
\hline$I_{x 3}=2.184$ & $I_{y 3}=0.2333$ & $I_{z 3}=2.2493$ & $\mathrm{Kg}^{*} \mathrm{~m}^{2}$ \\
\hline$r_{0}=0.6$ & $r_{1}=0.4$ & $r_{2}=0.3$ & $\mathrm{~m}$ \\
\hline$l_{0}=1.2$ & $l_{1}=0.8$ & $l_{2}=0.6$ & $\mathrm{~m}$ \\
\hline$m_{1}=100$ & $m_{2}=90$ & $m_{3}=70$ & $\mathrm{~kg}$ \\
\hline
\end{tabular}

We can obtain three differential equations of joint angles (as eqn. 21 shows) when we substitute simulation parameters into eqn. 20.

$$
\begin{aligned}
& \chi_{1} \ddot{\theta}_{1}+\chi_{2} \dot{\theta}_{1} \dot{\theta}_{2}+\chi_{3} \dot{\theta}_{1} \dot{\theta}_{3}=0 \\
& -16.8 s_{3} \ddot{\theta}_{3}-16.8 c_{3} \dot{\theta}_{3}^{2}-\chi_{2} \dot{\theta}_{1}^{2}-33.6 s_{3} \ddot{\theta}_{2}-33.6 c_{3} \dot{\theta}_{2} \dot{\theta}_{3}+2 \Gamma_{112} \ddot{\theta}_{1}+2 \eta_{1} \dot{\theta}_{1} \dot{\theta}_{2}+ \\
& 2 \eta_{2} \dot{\theta}_{1} \dot{\theta}_{3}+2+901.6 s_{2}+411.6 s_{23}=0 \\
& -\chi_{3} \dot{\theta}_{1}^{2}-16.8 s_{3} \ddot{\theta}_{2}+16.8 c_{3} \dot{\theta}_{2}^{2}+411.6 s_{23}=0
\end{aligned}
$$

where $\chi_{1}=-63.892 c_{2} s_{2}-16.632 c_{23} s_{23}-16.8 c_{23} s_{2}-33.6 s_{23} c_{2}$ $\chi_{2}=-63.892\left(c_{2}^{2}-s_{2}^{2}\right)-16.632\left(c_{23}^{2}-s_{23}^{2}\right)-50.4 c_{23} c_{2}-50.4 s_{23} s_{2}$ $\chi_{3}=-16.632\left(c_{23}^{2}-s_{23}^{2}\right)-33.6 c_{23} c_{2}-16.8 s_{23} s_{2}, \quad \eta_{1}=\frac{2 \partial^{2} M_{11}}{\partial \theta_{2}{ }^{2}}$, $\eta_{2}=\frac{2 \partial^{2} M_{11}}{\partial \theta_{2} \partial \theta_{3}}$

As can be seen from eqn. 21, the formula contains complex multi-variable, higher-order and nonlinear differential equations, so it is very difficult to solve to obtain an analytic solution. Therefore, we choose to use iterative methods. During the $0-t_{f}$ time, this time period is divided into $n$ small time periods, and the length of each time segment is $h$. We can transform eqn. 21 into an expression regarding angular acceleration of the three angles and obtain eqn. 22 based on the Taylor formula [12]:

$$
\begin{aligned}
& \mathrm{y}\left(x_{i+1}\right)=y\left(x_{i}\right)+h y\left(\dot{\mathrm{x}}_{i}\right)+\frac{h^{2}}{2} y\left(\ddot{\mathrm{x}}_{i}\right)+o\left(\mathrm{~h}^{2}\right) \\
& \mathrm{y}\left(\dot{\mathrm{x}}_{i+1}\right)=y\left(\dot{\mathrm{x}}_{i}\right)+h y\left(\ddot{\mathrm{x}}_{i}\right)+o\left(\mathrm{~h}^{2}\right)
\end{aligned}
$$


where we set an initial value $y_{A}=[0,95,156]$, and substitute $y_{A}$ into eqn. 21, then adjust variables according to eqn. 22 . When setting the final stop iteration angle value $y_{B}=[11.2,45.5,163$. $5], t_{f}=8 \mathrm{~s}$, and limited velocity and acceleration values are between $[-v, v],[-a, a]$ respectively. We can obtain a group of optimal discrete points consisting of three joint angles (as shown in fig. 3,fig. 4 , and fig. 5).

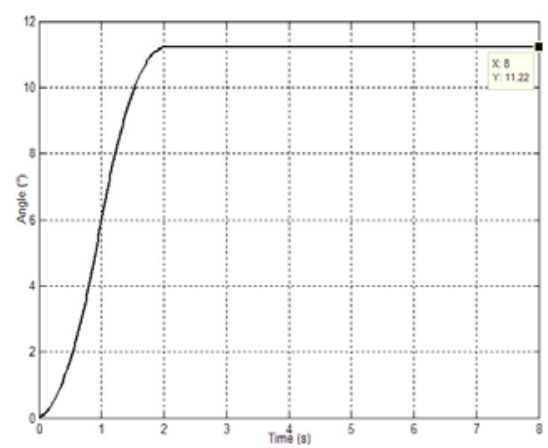

FIGURE III. ANGLE CURVE OF JOINT ANGLE 1.

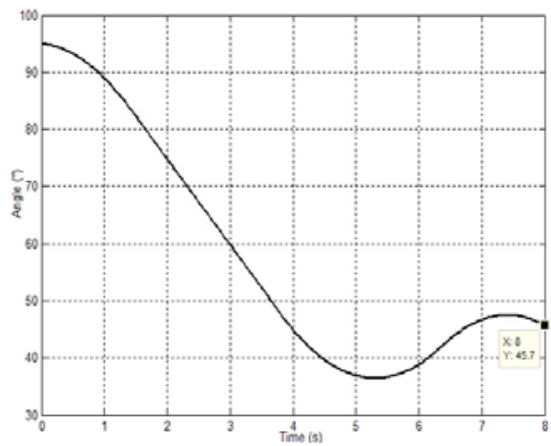

FIGURE IV. ANGLE CURVE OF JOINT ANGLE 2.

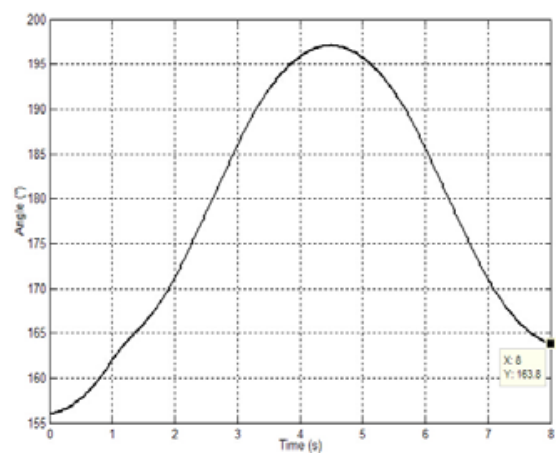

FIGURE V. ANGLE CURVE OF JOINT ANGLE 3.

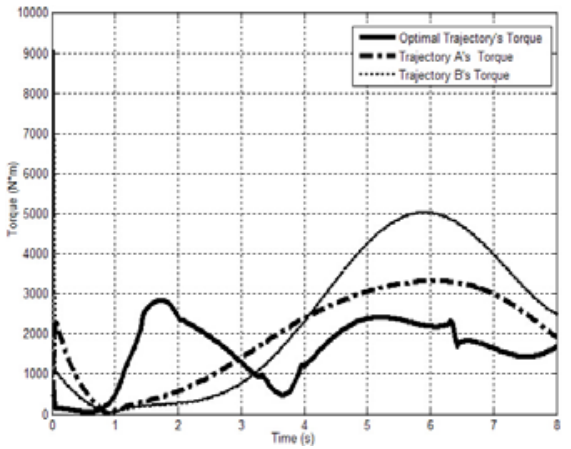

FIGURE VI. TIME-TOTAL TORQUE CURVE OF OPTIMAL TRAJECTORY FOR TRAJECTORY A AND TRAJECTORY B.

We arbitrarily set two angle trajectory curves A and B in which fitting with a cubic polynomial is used to compare with the optimal trajectory. Curve A three joint angles are $\theta_{A 1}=-0.016 \mathrm{t}^{3}-0.2313 \mathrm{t}^{2}+4.2767 \mathrm{t}$,

$\theta_{A 2}=0.3164 t^{3}-3.4731 t^{2}+1.3291 t+95, \quad$ and $\theta_{A 3}=0.39887 t^{3}-5.2562 t^{2}+17.5138 t+95$, respectively. At the same time, Curve $B$ three joint angles are $\theta_{B 1}=-0.1443 t^{3}+1.3437 t^{2}-0.11 t$,

$\theta_{B 2}=0.3345 t^{3}-4.3503 t^{2}+7.1947 t+95, \quad$ and $\theta_{B 3}=0.132 t^{3}-2.1895 t^{2}+10.0471 t+156$, respectively. The angles of the trajectories A and B are discretized, then substituted into eqn. 16 and eqn. 18 to obtain an optimal trajectory. The $\mathrm{A}$ and $\mathrm{B}$ time-total torque curve trajectories are shown in fig. 3.

By substituting the optimal trajectory, trajectory A and trajectory $\mathrm{B}$ into eqn. 18, the total torque during the setting time $0-t_{f}$ can be obtained respectively as $J_{\tau o}=4.7877 \times 10^{5}, \quad J_{\tau A}=5.9007 \times 10^{5}, \quad$ and $J_{\tau B}=6.9983 \times 10^{5}$, so we can conclude that the proposed optimal trajectory method can obtained a minimum torque and a consequent minimum energy consumption.

\section{CONCLUSION}

A robotic manipulator's energy consumption is mainly used to produce external torque. We minimize energy consumption by the study of the mathematical torque model of the manipulator then obtain an optimal trajectory, and proved the effectiveness of the proposed method in terms of energy savings by comparing it to other trajectories.

\section{ACKNOWLEDGMENT}

Foundation: Basic Research Project of Shenzhen (CXZZ20130322150432022), Technology Research Project of Shenzhen (JSGG20130923142607546)

\section{REFERENCES}

[1] Hirakawa, A.R., Kawamura, A., Trajectory generation for redundant manipulators under optimization of consumed electrical energy. Industry Applications Conference, 1996. Thirty-first IAS Annual Meeting, IAS '96, Conference Record of the 1996 IEEE, pp. 1626-1632, 1996.

[2] Hansen C, Oltjen J, Meike D, et al., Enhanced approach for energy-efficient trajectory generation of industrial robots. Automation Science and Engineering (CASE), 2012 IEEE International Conference on. IEEE, pp. 1-7, 2012.

[3] James T. Allison, Plant-Limited Co-Design of an Energy-Efficient Counterbalanced Robotic Manipulator Journal of Mechanical Design, 
135(10), pp. 101003-101003, 2013.

[4] Chung, Wing Kwong, Xu, Yangsheng, Path planning algorithm for space manipulator with a minimum energy demand. Robotics and Biomimetics (ROBIO), 2012 IEEE International Conference on. IEEE, pp. 1556-1563, 2012.

[5] Abhishek Agrawal, Sunil K. Agrawal, An energy efficient manipulator design approach: Application to a leg in swing phase. Journal of Mechanical Design, 129(5), pp. 512-519, 2007.

[6] Amin Nikoobin, Reza Haghighi, Lyapunov-Based Nonlinear Disturbance Observer for Serial n-Link Robot Manipulators. Journal of Intelligent and Robotic Systems. 55(2-3), pp. 135-153, 2009.

[7] Ogasawara, S., Hiramoto, K., Doki, H., Global optimal design of dynamic parameters of robot manipulators. Control Applications, 2007. CCA 2007. IEEE International Conference on. IEEE, pp.307-312, 2007.

[8] Jeon, Hong, Eslami, M., A minimum time joint-trajectory planning for industrial manipulator with input torque constraint. Robotics and Automation. Proceedings. 1986 IEEE International Conference on. IEEE, pp. 559-564, 1986.

[9] Meike D, Pellicciari M, Berselli G., Energy efficient use of multirobot production lines in the automotive industry: Detailed System Modeling and Optimization. Automation Science and Engineering, IEEE Transactions on.11.3 pp. 798-809, 2014.

[10] Faris W F, Ata A A, Sa'adeh M Y., Energy Minimization Approach for a Two-link Flexible Manipulator. Journal of vibration and control. pp. 497-526, 2009.

[11] Murray R.M., Li, Z., Sastry S.S., et al., A mathematical introduction to robotic manipulation. CRC press, 1994.

[12] Yanfang Li, Xianghu Liu, A learning algorithm and model of Taylor's formula based on functional network. Innovative Computing \& Communication, 2010 Intl Conf on, and Information Technology \& Ocean Engineering, 2010 Asia-Pacific Conf on (CICC-ITOE). IEEE, pp. 229-232, 2010. 